\title{
Managing PBC: Expanding the Provider Comfort Zone
}

\author{
David M. Chascsa ${ }^{1} \cdot$ Keith D. Lindor ${ }^{1,2}$ \\ Published online: 9 June 2018 \\ (c) Springer Science+Business Media, LLC, part of Springer Nature 2018
}

Of the causes of hepatic cirrhosis, primary biliary cholangitis (PBC) is among the most uncommon, caused by the inflammatory destruction of small intrahepatic bile ducts by an incompletely understood autoimmune process. Diagnostic criteria include two of the following three factors: persistent alkaline phosphatase elevation, detectable antimitochondrial antibody, and diagnostic liver biopsy [1]. Outside academic practices, given its low prevalence, PBC comprises only a small proportion of the patient panels of gastroenterologists and hepatologists, potentially creating unease among providers regarding management. Treatment is generally straightforward when using as sole therapy the first-line drug ursodeoxycholic acid (UDCA). For patients responding inadequately, obeticholic acid (OCA), a potent and potentially toxic synthetic FXR ligand, was approved by the US Food and Drug Administration in 2016, and its use was supported by treatment data generated from multiple therapeutic trials [2-4]. Furthermore, other second-line therapies such as fibrates and rituximab have reported success in clinical trials, further expanding PBC therapeutic options. Familiarity with the assessment of biochemical response to UDCA utilizing one of the many available criteria is essential to determining which patients warrant the risk of exposure to second-line therapy or consideration for study enrollment $[5,6]$.

In this issue of Digestive Diseases and Sciences, Jopson and colleagues reported the analysis of 6000 surveys sent to US providers defining themselves as gastroenterologists or hepatologists working outside academic practices [7]. The first 100 responses received from each group were analyzed. Using a Likert scale, respondents were asked to self-assess

\footnotetext{
David M. Chascsa

Chascsa.David@mayo.edu

Keith D. Lindor

Keith.Lindor@asu.edu

Mayo Clinic, Arizona, USA

2 Office of University Provost, Arizona State University Arizona, Tempe, USA
}

their competency regarding PBC management as well as their own implementation of best practice for PBC treatment and monitoring. Specifically, they were asked questions about their understanding and comfort with the use of UDCA, monitoring and treatment response, and cirrhosis management.

The study results highlight a significant discomfort among gastroenterologists and hepatologists with the management of PBC. PBC accounted for less than $20 \%$ of the providers' patient panels. $100 \%$ of hepatologists screened at-risk patients compared with 59\% of gastroenterologists, thus suggesting a missed opportunity to initiate treatment. Yet, even among hepatologists, only $72 \%$ felt highly competent in screening. While the majority of gastroenterologists and hepatologists understood the importance of early diagnosis and treatment, only $65 \%$ of gastroenterologists felt highly competent in so doing. Perhaps the most striking is the discrepancy between provider groups with regard to assessment of treatment response. While there were significant differences between hepatologists and gastroenterologists with regard to implementation of measures to assess treatment response (76 vs. $42 \%$ ), only an approximate third of hepatologists and gastroenterologists felt highly competent; there was statistically an insignificant difference. This observation highlights an important gap that could lead to adverse patient outcomes and suboptimal care. The majority of both groups implemented screening measures for cirrhosis, though significantly less gastroenterologists felt highly competent.

The strengths of Jopson's study are multiple: responses from 200 providers were analyzed, representing a large sample size; the study is unique in that it compares two groups of providers (gastroenterologists and hepatologists), groups which may often be lumped together; and while PBC accounts for a small percentage of a gastroenterologist's or hepatologist's overall practice, these providers working in non-academic settings will often manage the majority of affected patients. This study clearly indicates their unease, perhaps because $\mathrm{PBC}$ is a rare disease. 
The study underscores the need for increasing provider comfort with the treatment and assessment of treatment response of patients with PBC. Until 2016, comfort with treatment was of less concern as there was only one available therapy, UDCA. Now, OCA is available, providing an adjunctive or alternative for the $30 \%$ of patients with inadequate response or intolerance to UDCA. Still, providers must be adequately educated on the appropriate dosing, as adverse events related to inappropriate dosing have already been reported. Nevertheless, providers need familiarity and comfort using first-line treatments. The study suggests that neither gastroenterologists nor hepatologists are universally using UDCA and that there was no significant difference between the implementation of UDCA between gastroenterologists and hepatologists. The study was unable to assess why UDCA was not used universally.

This study is of interest in that it attempts to target a study population of non-academic providers in the USA. While the authors conclude that they reached an adequate sample size, they only analyzed 200 of a potential 6000 responses, accounting for only $3 \%$ of the distributed surveys. They do not otherwise report a response rate for received, completed surveys. Bias may have been introduced with earlier responders feeling more comfortable with PBC management. Nonetheless, if this were the case, the strength of their argument would only be amplified. Additionally, they do report reaching data saturation. The definition of an "hepatologist" in the USA has changed in the last 20 years: While initially it connoted a self-described interest in the practice of hepatology, reflecting in part the mandated 25\% requirement of current GI fellowship curricula for hepatology-related education and practice, it was not until 2006 that hepatology became an official subspecialty practice requiring additional training with separate board certification. Non-academic hepatologists are likely to become a rare breed as the majority of hepatologists are linked to academic transplant centers. This study relied on self-reporting of specialty; it is not clear from the paper whether the hepatology providers were certified in transplant hepatology, were gastroenterologists with an interest in hepatology, were internists with a special interest in hepatology, or were possibly advanced care practitioners. Regardless, it is concerning that UDCA was not universally used in PBC patients and only an approximate third of hepatologists and gastroenterologists felt competent to assess response to UDCA. A possible confounder is that there may have been referral bias with hepatology providers caring for patients with more complicated $\mathrm{PBC}$, and perhaps managing patients who have intolerance or suboptimal response to UDCA. This would not explain why such a low percentage of hepatologists felt competent to assess treatment response.

In conclusion, stratified therapy for $\mathrm{PBC}$ is now a reality. Optimal care relies on provider comfort with the available treatments and with assessment of treatment response. Currently, UDCA and OCA exist as treatment options in combination or as monotherapy. Both are associated with biochemical improvement and conversely with toxicities when dosed inappropriately. Future studies examining the link between provider comfort and improvement in patient outcomes are warranted. Providers need to be aware of investigational studies as well to identify patients needing referral for enrollment in clinical trials.

\section{References}

1. Lindor KD, Gershwin ME, Poupon R, et al. Primary biliary cirrhosis. Hepatology. 2009;50:291-308.

2. Jones D, Kowdley K, Chapman R, et al. OP02 The first new monotherapy therapeutic PBC study in a decade? An international study evaluating the farnesoid $\mathrm{X}$ receptor agonist obeticholic acid in PBC. Gut. 2011;60:A50.

3. Hirschfield GM, Mason A, Luketic V, et al. Efficacy of obeticholic acid in patients with primary biliary cirrhosis and inadequate response to ursodeoxycholic acid. Gastroenterology. 2015;148:751-761.

4. Nevens F, Andreone P, Mazzella G, et al. A placebo-controlled trial of obeticholic acid in primary biliary cholangitis. $N$ Engl $J$ Med. 2016;7:631-643.

5. Pares A, Caballeria L, Rodes J. Excellent long-term survival in patients with primary biliary cirrhosis and biochemical response to ursodeoxycholic Acid. Gastroenterology. 2006;130:715-720.

6. Corpechot C, Abenavoli L, Rabahi N, et al. Biochemical response to ursodeoxycholic acid and long-term prognosis in primary biliary cirrhosis. Hepatology. 2008;48:871-877.

7. Jopson L, Khanna A, Peterson P, et al. Are clinicians ready for safe use of stratified therapy in primary biliary cholangitis (PBC)? Dig Dis Sci. (Epub ahead of print). https://doi.org/10.1007/s1062 0-018-5074-8. 